\title{
Real time control of the ASBGo walker through a physical human-robot interface
}

\author{
Maria Martins ${ }^{\mathrm{a}, *}$, Cristina Santos ${ }^{\mathrm{a}}$, Anselmo Frizera ${ }^{\mathrm{b}}$, Ramón Ceres ${ }^{\mathrm{c}}$ \\ ${ }^{a}$ Industrial Electronics Department, University of Minho, Guimarães, Portugal \\ ${ }^{\mathrm{b}}$ Electrical Engineering Department, Federal University of Espirito Santo (UFES), Av. Fernando Ferrari, 514 Vitoria-ES, Brazil \\ ${ }^{\mathrm{c}}$ Bioengineering Group, Spanish National Research Council (CSIC), Crta. Campo Real km 0.200, Arganda del Rey, Madrid, Spain
}

\section{A R T I C L E I N F O}

\section{Article history:}

Received 2 February 2013

Received in revised form 15 October 2013

Accepted 18 October 2013

Available online 5 November 2013

\section{Keywords:}

Assistive mobility

Smart walkers

Fuzzy control

\begin{abstract}
A B S T R A C T
In this work it is presented the development of the conceptual design, implementation and validation of a Smart walker with an inexpensive integrated interface. This interface is based on a joystick and it intends to extract the user's command intentions. Preliminary sets of experiments were performed which showed the sensibility of the joystick to extract navigation commands from the user. These signals presented a higher frequency component that was attenuated by a Benedict-Bordner $\mathrm{g}$-h filter. The resulting interaction signals are then classified and converted into motor commands through a fuzzy logic controller. Additionally, the detection of possible falls and instability of the user was also one of the aims integrated onto the overall system architecture. Results have shown that the resultant movement of the walker was constant and safe without bumps. Short enquiries to the users have provided positive feedback about the device maneuverability in terms of easiness to use.
\end{abstract}

(c) 2013 Elsevier Ltd. All rights reserved.

\section{Introduction}

Mobility is a fundamental requirement for our daily life. The number of people with reduced mobility capabilities increases from year to year [1]. Thus, it becomes relevant to find tools to extend independent living and promote improved health. Recent advances in robotics have been developed for the elderly and lower limb disabled people. Examples include orthoses, canes, and Smart walkers.

Smart walkers [2-12] are intended to provide increased support and assistance during gait. Generally, Smart walkers have an integrated assistive navigation system and sensors to obstacles detection [2,3]. There is a concern to allow a stable gait through different handlebar designs [3,5], and by locating the electronics and other heavy components in

\footnotetext{
* Corresponding author. Tel.: +351 965774086.

E-mail addresses: mariam@dei.uminho.pt (M. Martins), cristina@ dei.uminho.pt (C. Santos), anselmo@ele.ufes.br (A. Frizera), ceres@iai. csic.es (R. Ceres).
}

the lower base of the walkers, thus improving the global passive stability and balance of the group walker-user.

In the field of Smart Walkers, researchers have been studying human-machine interaction, through the interpretation of user's movement intentions and transform this knowledge into motor commands (direction and velocity). This research area has recently witnessed a huge interest in searching for interfaces that can be intuitive [6] such that users do not need to realize the intelligent agent behind the driving wheel.

In [5] an intuitive force sensor interface was proposed to infer the navigation intents of the user. Despite the good results, users may present asymmetries during their gait that lead to different patterns of forces to the same intentions.

Despite these advances, recent studies on walker interfaces [4, 7-12] have not focused on the characterization of the signals gathered by the interface sensors, and it is currently lacking an exhaustive analysis of the main parameters involved in the interaction between the user and the device. It is still required to identify these parameters 
and their connection to the subsequent algorithms used for recognition and estimation. In [5] algorithms were developed to significantly attenuate or eliminate the components of the force signals related to the oscillations of the trunk, achieving, thus, the intentions of the subject on the guidance of the walker.

We propose to extend this work [5] and acquire the interaction signals through a novel and low cost humanmachine interface. Therefore, instead of the force sensors normally used, these were replaced by a joystick sensor mounted on the walker upper structure and the corresponding electronics. The proposed device is different from the one presented by Hashimoto [7] because the guiding of the walker is performed with the two hands, and additionally there is a support for the forearms, thus making the proposed system safer, more stable and intuitive.

In our work, the human-machine interaction is characterized according to experiments performed without any motorization of the device in order to capture the natural signals that represent the real movement intentions of the user. In case any undesired component is detected, real time signal processing algorithms eliminate those components. This provides for a tuning of the developed algorithms such that these are also able to characterize the interaction between the walker and the user in real time experiments in which the walker has the motors on. Finally, a motion control algorithm based on Fuzzy logic, controls the direction and velocity of the walker accordingly.

Smart walkers usually do not address safety issues considering risks of falls or other mishaps that can arise while the user is guiding the walker. These issues are also tackled in this work, and integrated onto the overall system architecture.

The feasibility of the proposed device is verified by performing a series of experiments with healthy users. Results show that despite the small variations of the acquired joystick signals, the resultant movement of the walker was constant and safe without bumps. The proposed control strategy is able to provide for a smooth driving, with fast response and no sense of delay. Short enquiries to the users have provided positive feedback about the device manoeuvrability in terms of movement resistance and easiness to use.

This paper is organized as follows. Section 2 describes the design and overall system architecture of the proposed Smart walker. Section 3 presents the sensor device in detail. The applied signal processing strategy to extract the undesired signal components from the acquired signals is presented in Section 4. Section 5 presents and discusses the developed control strategy based on a fuzzy logic system. Section 6 presents the safety considerations. The achieved results are presented in Section 7. Finally, conclusions are discussed in Section 8.

\section{ASBGo walker}

The work herein described is part of a Smart walker project which ultimate goal is to improve the safety and stability of walkers, thus contributing and reinforcing to the maintenance or to the improvement of the physical and cognitive capabilities of the user, through functional compensation. This paper presents the development of the conceptual design, implementation and validation of a Smart walker with an inexpensive integrated device intended to extract the users' command intentions. It was designed to be user-friendly and efficient, meeting usability aspects, but not being demanding at the user cognitive level.

The proposed Smart Walker for mobility Assistance and monitoring System Aid (ASBGo) is presented in Fig. 1. This robotic walker was built during this work, as well as its electronics, through the mechanical modification of a conventional four-wheeled walker. Among the different types of walkers it was selected a traditional four-wheeled that allows the installation of the electronics and heavy components in the lower zone of the walker to improve the general stability of the set user-walker. An additional structure was implemented to integrate the motors of the smart walker and an additional support base for the upper limbs was also included, in order to find the best way to frame the proposed device.

Fig. 1 depicts the overall architecture of the proposed system. In the long run, this project targets a commercial focus. Therefore, it is important to rely on more inexpensive Human-Machine interfaces. The proposed device was implemented with simple and low cost sensors. It is based on a joystick, block Interaction Device in Fig. 1, for the acquisition of the user's gestures during assisted gait. A subsequent real time signal processing strategy, block Signal Processing, interprets and characterizes these signals. Such interaction signals are then classified and converted into motor commands through a fuzzy logic controller, block Control Strategy. The possibility to detect possible falls and instability of the user was also one of the aims integrated in this project, block Safety.

The joystick and the other integrated sensors were implemented into a real time architecture based on an Arduino Platform, as it is an economic approach. In Fig. 1 the laptop is only used for data store issues for off-line analysis, i.e. it is not part of the system architecture.

\section{Interaction device}

In order to provide for safe guidance of the walker, in which the user controls the device in accordance with his gestures, the proposed ASBGo device has to capture the user's directional intent correctly. In order to do so, this intent will be identified from physical manipulation of the walker handles; since user's directional intent and his physical manipulation usually are mutually consistent.

The proposed device consists on placing, at the center of the upper base support, a joystick (hall-effect, HFX-33S12034) associated with two springs (Fig. 2a). The joystick is a robust and low cost device that does not require excessive use of electronics, and reduces the risk of failure. As this system is embedded, it facilitates a simple and compact upper structure design.

The development of this device took into account that walking is a complex combination of omni-directional 


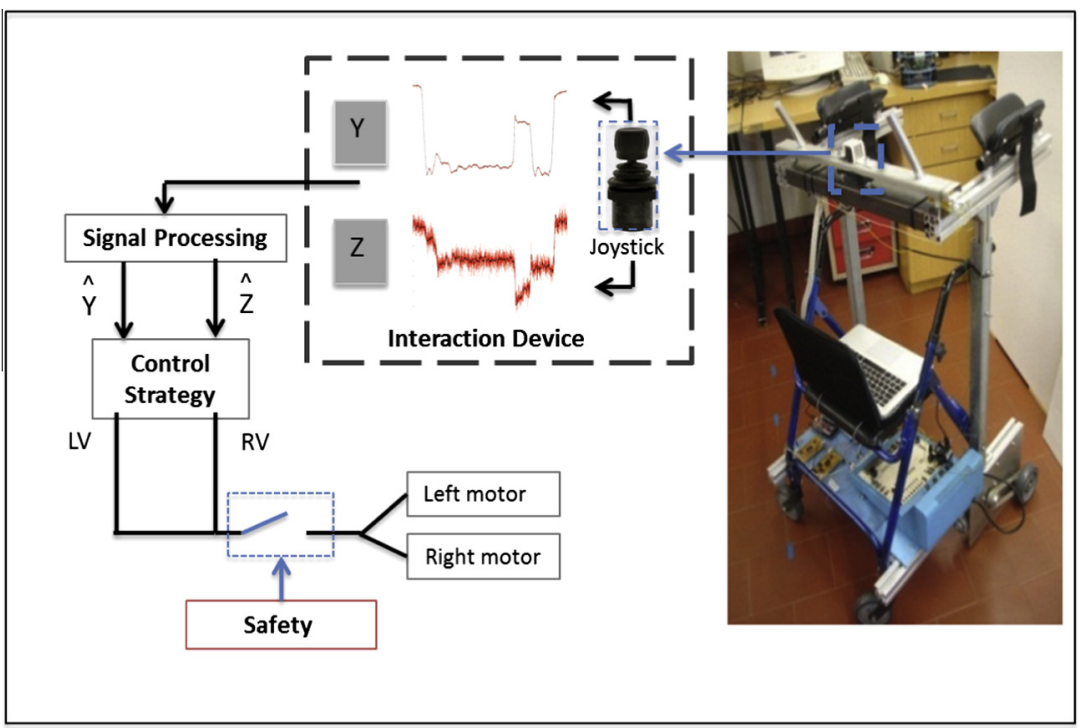

Fig. 1. System Architecture. LV: Left Velocity; RV: Right Velocity. Safety box includes the sensors and algorithms developed for safety considerations.

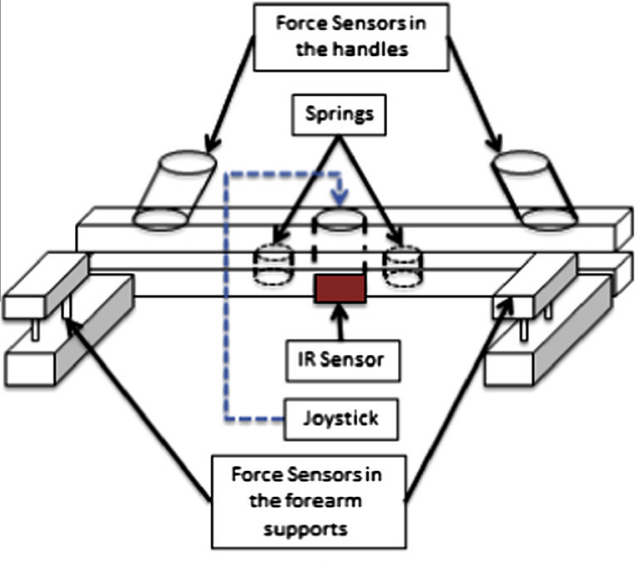

(a)

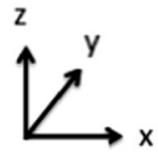

(b)

Fig. 2. (a) Schematic of the ASBGo interface with the joystick and other safety complement sensors and (b) schematic of the joysticks' displacement.

motions, including forward and rotations' movements. The device should have the capability to "read" and interpret all these kind of intended motions, to follow the user's movement, and to provide for a good walking support. Moreover, the user subconsciously manipulates the device in order to set directional instructions.

Functionally, the interaction device works as follows. When the user begins to walk, he applies grip forces/torques to the handles. At this moment, the upper base structure slightly moves according to the direction of these forces. As this structure is mechanically coupled to the joystick, the joystick suffers a slight displacement that characterizes the user directional intent. Hence, the user's efforts are successfully converted into small displacements of the joystick. In addition, as the horizontal stiffness of the springs is lower than the vertical one, the joystick can detect the forward force and torque components with high accuracy even if a large vertical force is applied. Another reason for having the springs is that the joystick must return to the rest position when the user leaves the handles.

The joystick outputs three different signals $(X, Y, Z)$, measured in Volts, that describe the imposed movement in the $X Y Z$-axis attached to the joystick (Fig. 2b). In this work, only the $Y$ and $Z$-signals are used, i.e. it will be only evaluated the forces applied in the $Y$ and $Z$ direction.

Signal $Y$ describes the angular rotation around the $X$ axis and represents the straight or forward movement intention of the user in the $Y$-direction. Signal $\mathrm{Z}$ is the angular rotation around the $Z$-axis and represents the curvilinear movement intention of the user (intent to turn left/right).

\section{Signal processing strategy}

The Joystick signal can be affected by the vibrations of the structure (irregularities of the ground), electromagnetic 
noise and wheel eccentricities. Thus, the noise components must be eliminated in real-time, not causing a considerable delay on the signal.

The higher frequency components can be eliminated with forth and back recursive digital filters, such as Butterworth filters, without causing phase distortion. However, this approach is not real-time implementable.

A low computational cost is needed to avoid use of expensive hardware to process the signal and considering power consumption and the possibility of introducing temporal delay on the filtered signal. When the filtered signal is used to control the walker, the user should not perceive the delay between his commands and the movement of the walker. The human perception threshold in applications like this is known to be around the $200 \mathrm{~ms}$ [16], and this will be the employed reference.

In the literature [13], two types of filters were identified to be frequently used as potential candidate algorithms to eliminate components of higher-frequency in real-time. These filters are the $\mathrm{g}-\mathrm{h}$ filter and the Kalman filter, and they are usually called of tracking filters.

\section{1. $g$-h Filter}

The $\mathrm{g}-\mathrm{h}$ filter is a simple recursive filter given by the following formulae [13]:

$\hat{\dot{x}}_{k, k}=\hat{\dot{x}}_{k, k-1}+\frac{h_{k}}{T_{s}}\left(y_{k}-\hat{x}_{k, k-1}\right)$

$\hat{x}_{k, k}=\hat{x}_{k, k-1}+g_{k}\left(y_{k}-\hat{x}_{k, k-1}\right)$

$\hat{\dot{x}}_{k+1, k}=\hat{\dot{x}}_{k, k}$

$\hat{x}_{k+1, k}=\hat{x}_{k, k}+\hat{\dot{x}}_{k, k} T_{s}$

where $T_{s}$ is the time between time steps.

The filtering Eqs. (1) and (2), estimate the current position, $\hat{x}_{k, k}$, and velocity, $\hat{\dot{x}}_{k, k}$, based on previous predicted position, $\hat{x}_{k, k-1}$, and velocity, $\hat{\dot{x}}_{k, k-1}$, taking the current measurement, $y_{k}$, to account. In our case, this measurement corresponds to the $Y$ and $\mathrm{Z}$-signals. Assurance on measures is weighted by gains, $g_{k}$ and $h_{k}$.

The prediction Eqs. (3) and (4) predict the future estimated position, $\hat{x}_{k+1, k}$, and velocity, $\hat{\dot{x}}_{k+1, k}$, based on a first order dynamic model of the process. As $\mathrm{g}-\mathrm{h}$ trackers consider a constant velocity model, predicted velocity is equal to the current one. This assumption is reasonable since human movements are slow.

Parameters $g_{k}$ and $h_{k}$ have to be tuned prior to operation of filter. If these parameters are constant, for each $y_{k}$ only two storage variables are required, one for the latest predicted first derivative $\hat{\dot{x}}_{k, k}$ and one for the latest predicted state.

To select the filter gains $(g, h)$, we consider the Benedict-Bordner Filter (BBF) [13] and the Critically Dampened Filter (CDF) [13].

BBF minimizes the total transient error, defined as the weighted sum of the total transient error and the variance of prediction error due to measurement noise errors [13]. The BBF is the constant $\mathrm{g}-\mathrm{h}$ filter that satisfies: $h=\frac{g^{2}}{2-g}$

This equation relates $g$ and $h$, such that the BBF has only one degree of freedom. In $\mathrm{g}$-h filters increasing the value of $g$ diminishes the transient error, tracking higher frequencies.

CDF minimizes the least squares fitting line of previous measurements [13], giving old data lesser significance when forming the total error sum. This is achieved with a weight factor $\theta$. Parameters in the $\mathrm{g}-\mathrm{h}$ filter are related by:

$g=1-\theta^{2}, h=(1-\theta)^{2}$

\subsection{Kalman filter} by

The user's intentions first order dynamic model is given

$x_{k+1}=x_{k}+\hat{\dot{x}}_{k} T$
$\hat{\dot{x}}_{k+1}=\hat{\dot{x}}_{k}+u_{k}$

The equation that links the actual state $x_{k}$ and the measured $y_{k}$ is called the observation equation:

$y_{k}=x_{k}+v_{k}$,

where $v_{k}$ is the measurement noise and $u_{k}$ the random process noise.

It was assumed that the errors could be modeled using Gaussian Distributions. The Kalman filter parameters are the measurement noise covariance $R$, and the process noise covariance $Q$.

The measurement noise covariance $R$ is measured prior to operation of the filter [14]. This is practical since generally it is simple to take some off-line sample measurements in order to determine the average variance of the measurement noise, $\sigma_{u}^{2}: R=\left[\sigma_{u}^{2}\right]$. The determined value was of $8.82 \times 10^{-5} \mathrm{rad}^{2} \mathrm{~s}^{-2}$ and $1.3 \times 10^{-5} \mathrm{rad}^{2} \mathrm{~s}^{-2}$ for the $\mathrm{Y}$ and $\mathrm{Z}$-signal noise, respectively.

The selection of the process noise covariance $Q$ is generally more difficult [14]. In this application the process noise covariance $Q$ is formulated based on the first derivative noise, which affects the estimation of the user's command intentions. $Q$ is calculated using off-line measures of the signal. For each measure the covariance of the signal is calculated. Finally, the process noise covariance is the average of all the calculated covariances. A piecewise small constant first derivative model is considered [13]:

$Q=\left[\begin{array}{cc}\sigma_{v}^{2} & 0 \\ 0 & \sigma_{v}^{2}\end{array}\right]$,

where $\sigma_{v}^{2}$ is the variance of the random first derivative component of the process noise.

The Kinematic Estimation Error (KTE) was chosen to tune the filter constants. KTE evaluates the smoothness, response time, and execution time of a tracking algorithm [15] and is expressed by:

$K T E=\sqrt{|\bar{\varepsilon}|^{2}+\sigma^{2}}$ 
where $|\bar{\varepsilon}|^{2}$ and $\sigma^{2}$ are the mean and variance of the absolute estimation error between the desired and the measured signal. The desired signal is obtained by filtering offline the signals' measurements with a Butterworth filter.

\section{Control strategy}

In this section, it is described the Control Strategy block of Fig. 1. The two inputs are the $\hat{Y}$ and $\hat{Z}$ filtered signals, and they are combined to generate motor commands. It addresses a control strategy based on fuzzy logic to classify the signals sent by the joystick and transform them into motor outputs (direction and velocity), in such way that the motors are driven according to the user's intent.

The block entitled signal conditioning is better presented in Fig. 3. The $\hat{Y}$ and $\hat{Z}$ filtered signals are firstly amplified with a gain and normalized to $[-1,+1]$, according to the acquired signals during the first seconds of movement. This enables to a simple signal calibration of each patient. Finally, a dead-zone is included to prevent the motors to move when input signals are close to zero.

The premise behind fuzzy logic is that precise outputs can be obtained from imprecise or vague inputs [17]. For each joystick signal, a set of membership functions (MF) constituted by Gaussian and S-shaped functions were defined. After the fuzzy logic, the signal is also conditioned (block output conditioning, Fig. 3) through a small integration to avoid abrupt changes in the control signals and their range of values is also adjusted and sent to the motors' board.

\section{Safety}

A very important aspect of smart walkers is to provide for security/safety such that the user feels safe while controlling the walker. Otherwise, the user will not use this device and resort to others such as the wheelchairs.

The detection of users' falls while walking with the walker was one of the aims integrated on this work (module Safety in Fig. 1). The outputs of this Safety module have the highest priority and enable or not the walker movement.
In order to detect possible forward falls of the user, the approximation of the user to the walker is monitored through infrared sensing (GP2Y0A21YKOF) placed on the walker at the chest's height (Fig. 2a). If the user is falling forwards, the distance between the user's chest and the walker is decreased, and the infrared sensor output increases. In Fig. 4a it is depicted the IR signal of a walking user with the walker. At $t \approx 18 \mathrm{~s}$, the user falls. The IR signal decreases accordingly to the user approximation to the walker.

An algorithm was developed to detect abrupt changes on the signal, to then detect if the user was falling forward. When such situation is detected, the walker stops immediately.

In order to detect if the user is falling backwards three different procedures were considered. First, the walker cannot move backwards. So, if the user pushes the upper structure in his direction, the walker stops. Second, two force sensors were integrated on the handlebars (Fig. 2a) to detect if the user was grasping or not the handles. If the two handlebars are not being grasped, the walker immediately stops. Finally, two Flexiforce sensors ${ }^{\circledR}$ force sensors, one on each forearm support, are used to verify if the user is with his arms properly supported on the forearms supports (Fig. 2a). If the user relies on both supports/ grasps the handles, the measured force signal increases, and the walker is enabled to move. If the user is not loading the sensor the output signal decreases until it reaches zero (Fig. 4b) and the walker immediately stops. The same algorithm developed for the IR signal, was used in these situations.

Finally, the maximum speed that the walker can achieve was limited to $0.7 \mathrm{~m} / \mathrm{s}$, accordingly to the defined speed in the literature for elder [18].

\section{Results and discussion}

\subsection{Human computer interaction}

To extract the signals from the joystick, and to evaluate and verify its sensibility to detect the user intents, it was performed a study with eleven healthy volunteers, with

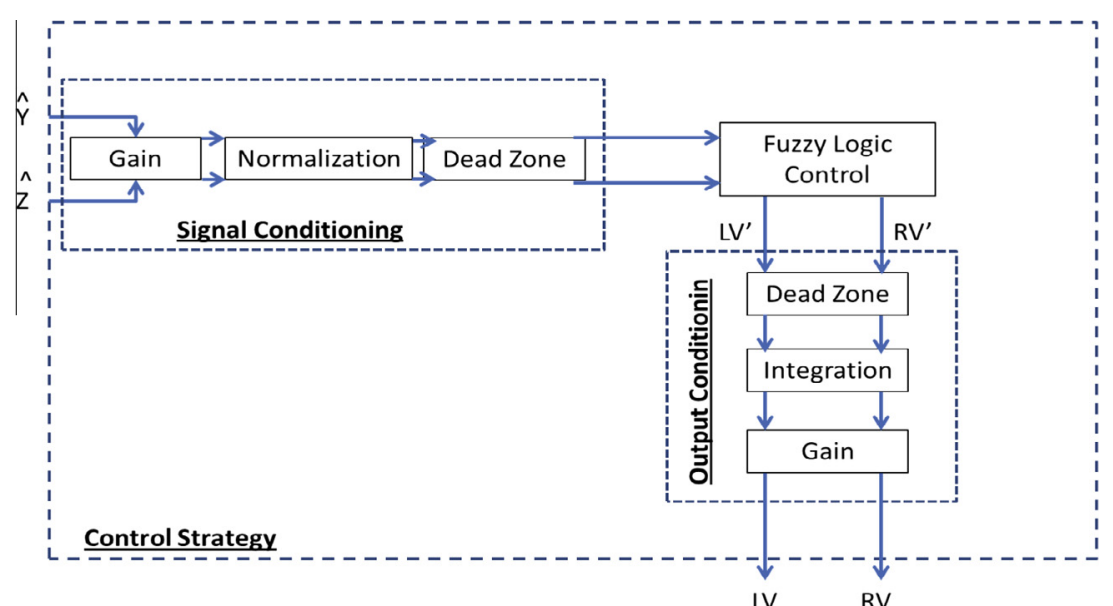

Fig. 3. Control strategy block. 
(a)

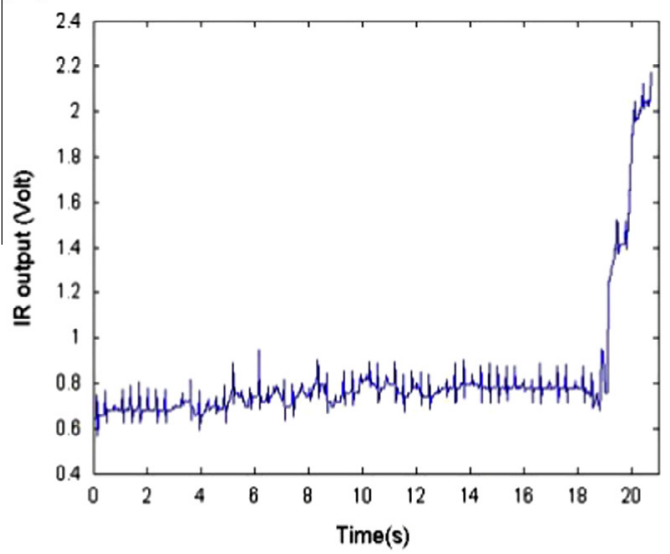

(b)

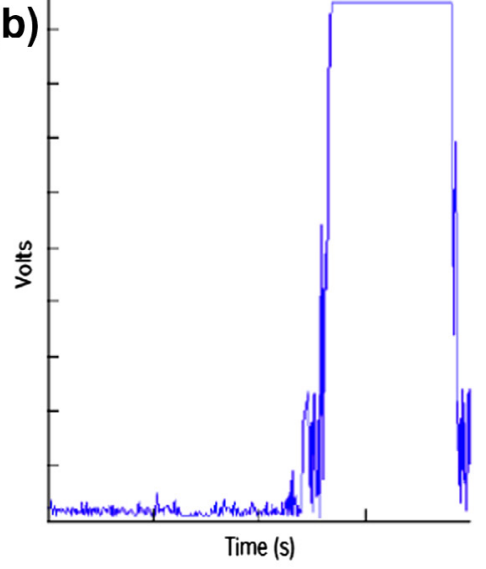

Fig. 4. (a) Typical IR output signal from an user that is walking normally and then falls forward, (b) typical signal of the Flexiforce sensor when first, the user is not loading the sensor, and then loads.

no history of dysfunctions on either upper or lower limbs. These volunteers had to perform three simple experiments like moving forward or/and then turn left or right (Fig. 5) and repeat them five times each. These experiments were performed without motorization of the walker for better transparency.

On each experiment, forward $(\mathrm{Y})$ and rotation $(\mathrm{Z})$ signals were acquired. The Y-signal gives the intention to move forward and according to the applied force, the signal will have more or less amplitude. The Z-signal, gives the intention to perform a curve and the signal will present high or low amplitude depending if the performed curve is more or less accentuated. The intention to turn right or left is detected by the sign of the signal, i.e. turn left causes negative signal and turn right causes positive signal.

Fig. 6 shows an example of typical $Y$ and $Z$ joystick data, corresponding to one trajectory performed with the device by one of the volunteers. The main steps of the trajectory consisted in: Stop (S1); Walk forward (S2); Turn left (S3); Walk forward (S4) and stop (S5).
Initially the user is stopped (S1) and both Y and Z-signals are zero. When the user begins to walk forward (S2), he pulls the handles and the Y-signal becomes negative. The Z-signal remains approximately zero, since the joystick is not rotated around the Z-axis when the user walks forward. In this time range, it can be observed that Y-signal drifts slightly as it approaches to step S3. This happens because the user is subconsciously breaking slowly the walker to change his direction intent. Further, the user usually does not apply the same force on both handles, and it can be noticed that the Z-signal changes a little bit when the user starts to walk forward.

When the user turns left (S3), the Y-signal increases to zero, and the $Z$-signal becomes negative, since the joystick also rotates around the $Z$-axis to the left side (in case the user turns right, the only difference is that the Z-signal becomes positive instead of negative).

Next, the user moves forward (S4). The Z-signal returns to zero and the Y-signal becomes negative. At the end of

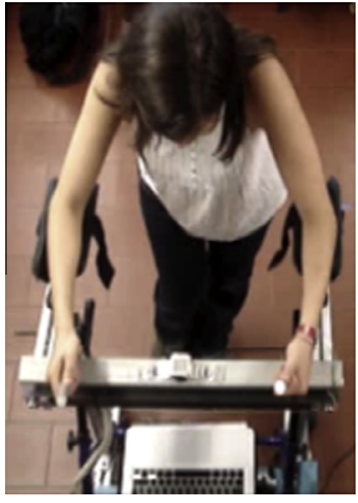

(a)

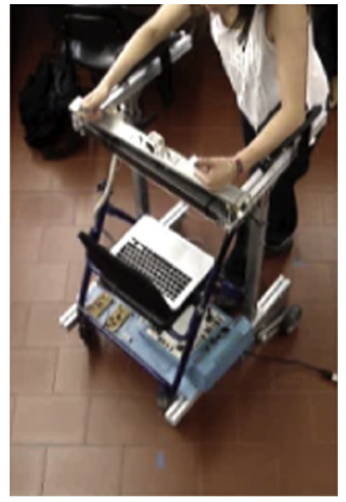

(b)

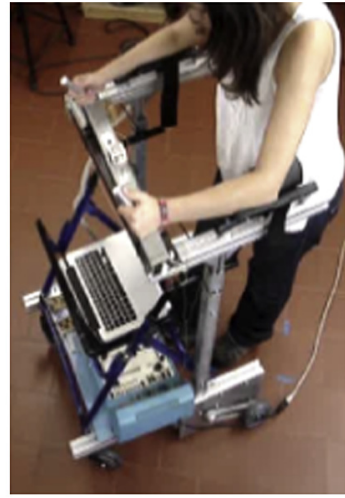

(c)

Fig. 5. (a) Walking forward, (b) turning left and (c) turning right. 


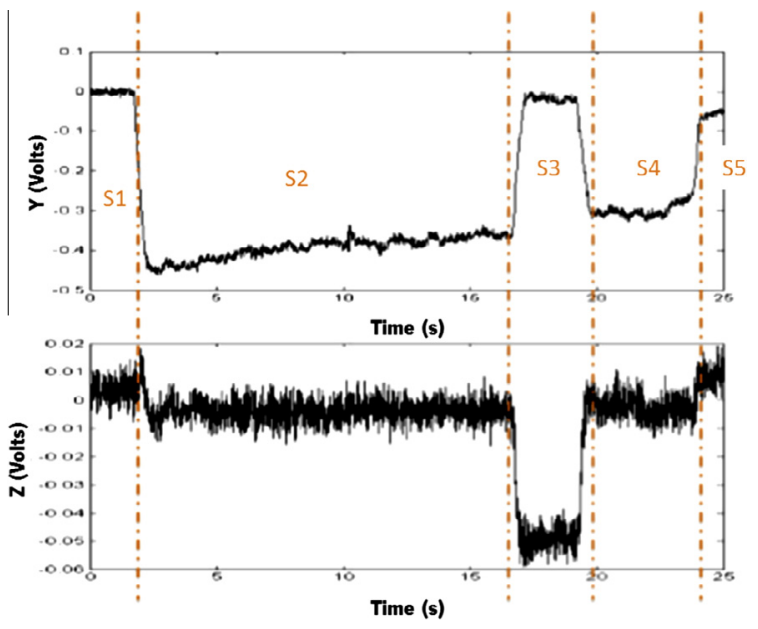

Fig. 6. Typical raw $\mathbf{Y}$ and $\mathbf{Z}$ joystick data in the ASBGo walker when the user is performing the following trajectory: S1 - The user is stopped, S2 User starts walking forward, S3 - User turns left, S4 - User walks forward and S5 - User stops walking.

the trajectory, the user stops (S5), and the Y-signal returns to zero.

The analysis of the acquired data with the volunteers, allows concluding that the joystick is sensitive enough to detect the user's intent to change his direction and velocity.

\subsection{Signal processing}

By observing the eleven acquired $\mathrm{Z}$ and Y-signals, two main components of the signals were identified. The collected data yields that the user's intent occurs in a frequency range between 0 and $2 \mathrm{~Hz}$ in both $\mathrm{Y}$ and $\mathrm{Z}-$ signals, and the higher-frequency components are related to noise.

Table 1 shows the signal-to-noise ratio (SNR) of these signals. The SNR of the Z-signal is much lower than the one of the Y-signal. This means that Z-signal has to be further filtered compared to the Y-signal.

Before testing the presented filters, it is first needed to select the BBF parameter, $g$, CDF parameter, $\theta$ and Kalman filter parameter, $Q$, from joystick data ( $Y$ and $Z$-signals). For tuning, it was used the joystick data from eleven healthy volunteers, performing three experiments each with five repetitions, as presented in Section 7.1.

These joystick data was introduced in each one of the filters algorithm (Kalman filter, BBF and CDF). In order to determine the best parameter value for each filter, a broad range of values was tested offline. Each filtered signal resulting from all parameter values inside the range, was processed according to KTE. The best solutions for each sig-

Table 1

SNR (signal-to-noise ratio) of the $\mathbf{Y}$ and $\mathbf{Z}$ signals.

\begin{tabular}{lll}
\hline Signal & $\mathrm{Y}$ & $\mathrm{Z}$ \\
\hline SNR & 48.98 & 2.37 \\
\hline
\end{tabular}

nal, i.e. the ones with the lowest KTE, were chosen, yielding a total of 165 KTE best solutions for each filter parameter. Finally, the 165 solutions for each parameter were averaged, as well as the average delay between the raw and the filtered signal for each parameter.

Table 2 presents the average values for $g, \theta$ and $Q$ parameters; the average KTE for each Y and Z-signals, and the average delay between the original $Y$ and Z-signals and the filtered one. Note that $g$ parameter of the Z-signal compared to the one of Y-signal shows a lower value. But the average $\theta$ and $Q$ parameters of the Z-signal compared to the ones of the Y-signal show a higher value. These results were as expected, since the SNR of the Z-signal is much larger than the one of the Y-signal, requiring to be further filtered [13].

The maximum value of delay was $36.2 \mathrm{~ms}$. Since human perception (200 ms) is larger than this delay, all the filters provided for largely inferior values and thus all filters are of high quality for a human-machine interaction.

The achieved KTE is very low for all filters, but the BBF detains the lower values for both the KTE, its dispersion and also the signal delay. Thus, it is the best option to choose for this application.

Figs. 7 and 8 illustrate a real example of $Y$ and $Z$ joystick signals, when applying BBF and CDF and BBF and kalman filters, respectively. In both pictures it is also shown the reference signal, i.e., the signal filtered with the Butterworth filter.

The BBF shows a higher attenuation on the oscillations than the CDF and Kalman filters. Thus, a Benedict-Bordner $\mathrm{g}$-h filter was applied to the joystick data. The $g$ parameter was chosen to be $44.29 \times 10^{-3}$ and $16.87 \times 10^{-3}$ for the $Y$ and Z-signal filters.

Table 3 depicts the average SNR of both signals. Note that there was an increase in the average SNR, meaning that the signals were filtered, i.e. the noise was attenuated. Although the SNR of the $\hat{Z}$-signal is $1 / 6$ of the one of the $\hat{Y}-$ signal, $\hat{Z}$-signal seems to be filtered enough to this application. However, a correct conclusion can only be achieved during the validation process (Section 7.3).

In summary, the BBF filter has a low computational cost implementation, and thus ideally applies to our application, since it can run in a low cost hardware while providing enough robustness for a commercial device.

The Y and Z-signals are filtered individually with BBF filters, and are used to drive the walker through a classifier based on fuzzy logic, as described in the following section.

\subsection{Control strategy}

In order to validate the proposed architecture, experiments were performed with the same eleven healthy users. The walker was motorized and the proposed fuzzy control system was functioning. The proposed system is expected to be user friendly, meaning it will help the user in performing the desired trajectories. Therefore, the users will have to qualify the walker according to the resistance it offers to the desired movement (forward and turning movement) and also according to the desired velocity.

Fig. 9 shows an example of the signals acquired of one user when he performed the same trajectories as during 
Table 2

Filter parameters based on the KTE and delay for the $\mathbf{Y}$ and $\mathbf{Z}$-signal. Table provides for mean \pm standard deviation.

\begin{tabular}{llcr}
\hline Signal & Parameter & Value & KTE $\left(\times 10^{-3} \mathrm{rad} / \mathrm{s}\right)$ \\
\hline $\mathrm{Y}$ & $g$ & $44.20 \pm 4.97\left(\times 10^{-3}\right)$ & $6.46 \pm 0.91$ \\
& $\theta$ & $0.974 \pm 3.85 \times 10^{-3}$ & $6.81 \pm 0.75$ \\
& $Q$ & $3.21 \pm 0.55\left(\times 10^{-7}\right)$ & $9.66 \pm 0.86$ \\
$\mathrm{Z}$ & $g$ & $16.87 \pm 2.51\left(\times 10^{-3}\right)$ & $2.93 \pm 1.99$ \\
& $\theta$ & $0.990 \pm 1.1 \times 10^{-3}$ & $2.99 \pm 0.11$ \\
& $Q$ & $3.26 \times 10^{-9} \pm 8.78\left(\times 10^{-9}\right)$ & $23.8 \pm 1.70$ \\
\end{tabular}
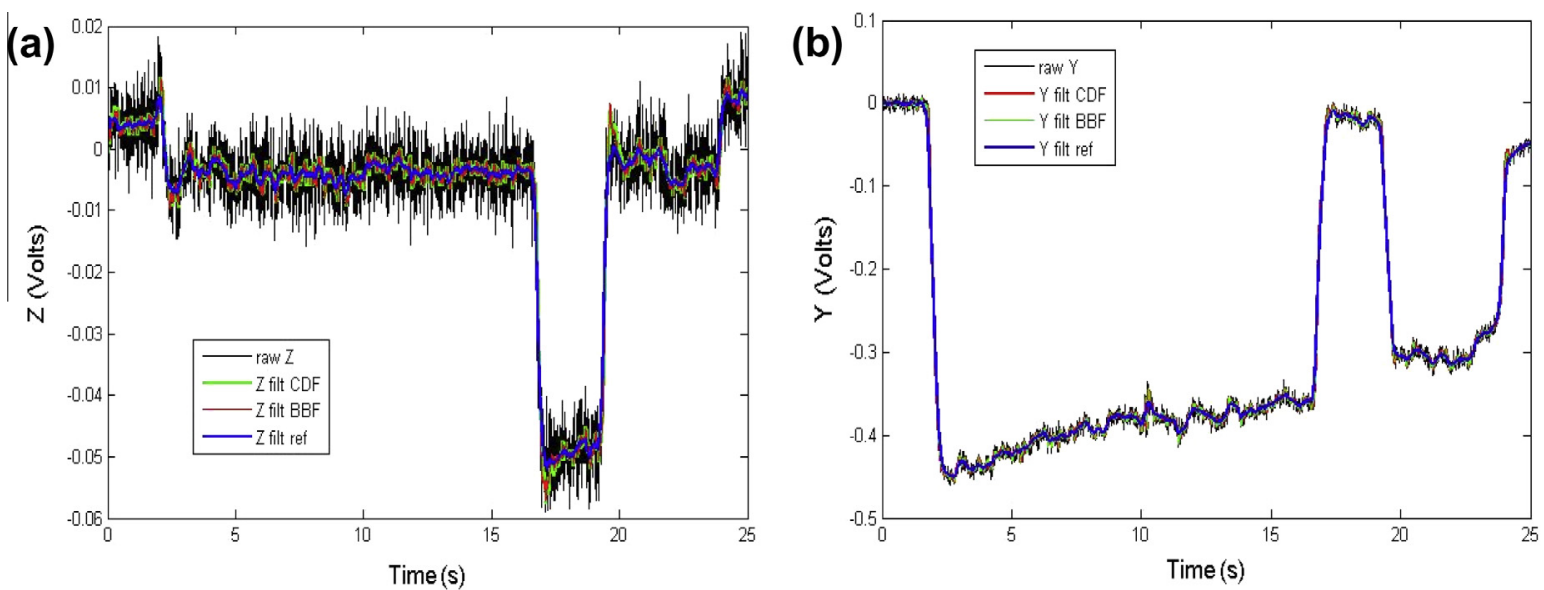

Fig. 7. Superposition of the raw joystick, (a) the superposition of the raw $\mathbf{Z}$ with the results of BBF, CDF and Butterworth and (b) the superposition of the raw $\mathbf{Y}$ with the results of BBF, CDF and Butterworth.
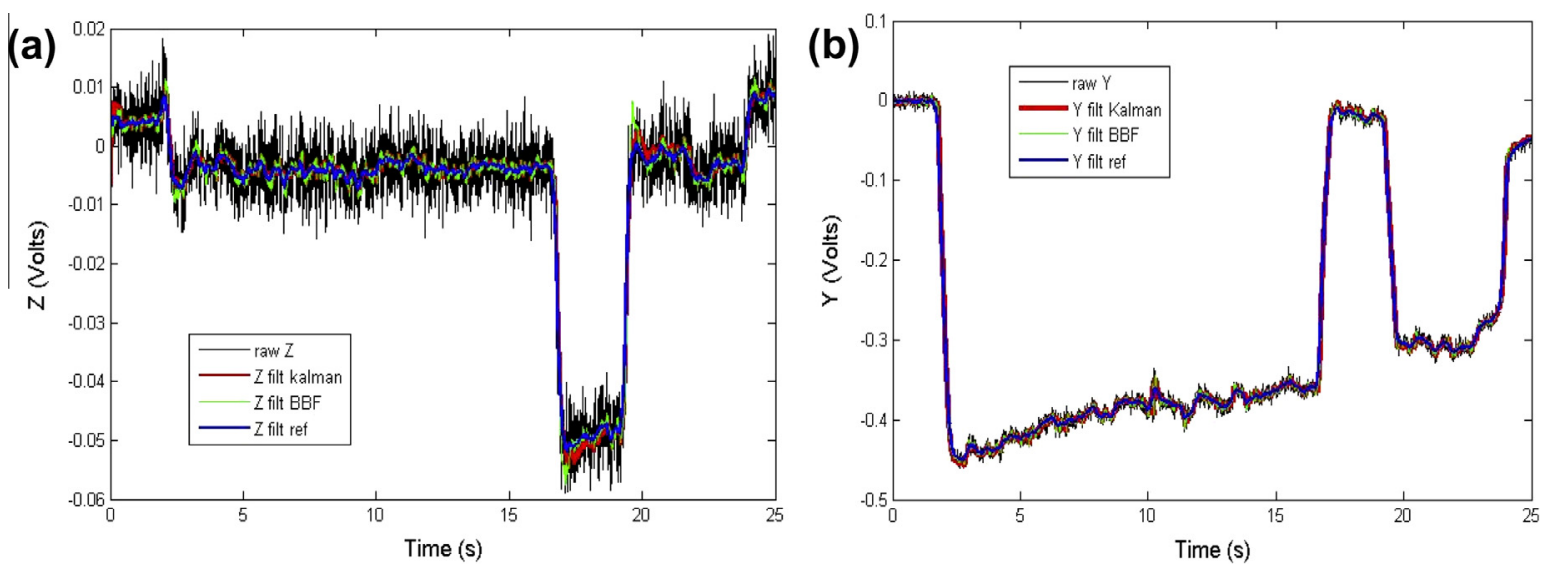

Fig. 8. Superposition of the raw joystick signal with the results of BBF, Kalman and Butterworth. (a) Raw Z-signal and (b) Raw Y-signal.

Table 3

SNR of the $\mathbf{Y}$ and $\mathbf{Z}$ signals filtered with the Benedict-Bordner filter.

\begin{tabular}{lll}
\hline Signal & $\hat{Y}$ & $\hat{Z}$ \\
\hline SNR & 50.05 & 7.77 \\
\hline
\end{tabular}

the development of the control and signal processing strategies: Stopped, walk forward (from $t \approx 1 \mathrm{~s}$ until), turn right (from $t \approx 9 \mathrm{~s}$ until $t \approx 13 \mathrm{~s}$ ), walk forward (from $t \approx 13 \mathrm{~s}$ ) and $\operatorname{stop}(t \approx 16 \mathrm{~s})$.
Fig. 9(a) depicts the raw joystick and BBF filtered signals acquired while the user was walking with the motorized walker. Note that despite the addition of the motors and the control strategy in generating the movement of the walker, these signals present the same features as the ones acquired with no motorization and control (Fig. 6). The only difference is that the $\mathrm{Y}$ and Z-signals, with the motors on, present a more accentuated noise. This is caused by the vibrations and electromagnetic noise due to the motors. However, as it is shown in these same figures, the results 

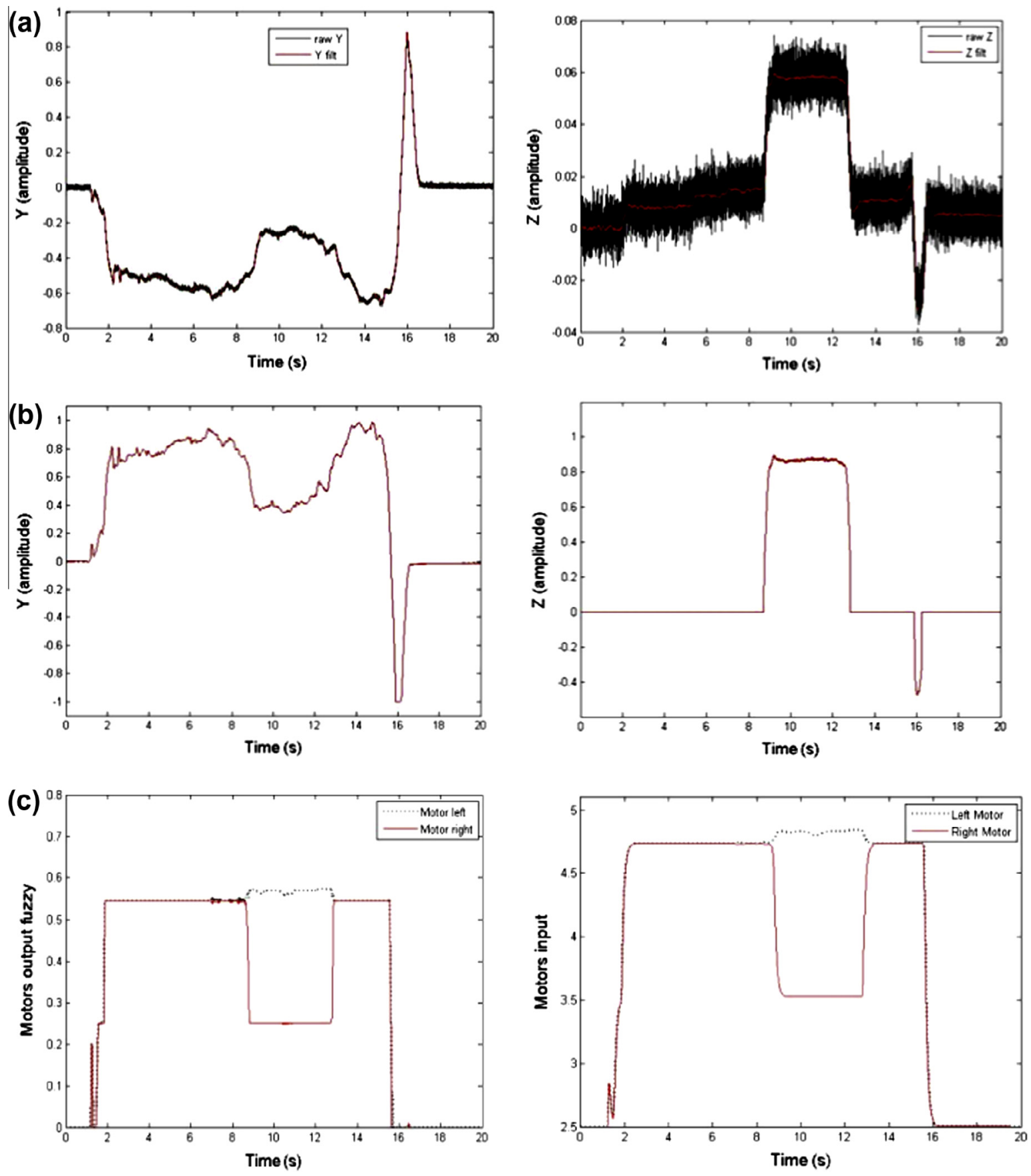

Fig. 9. Results from the system architecture of the ASBGo walker acquired while the user was walking with the device, (a) raw acquired joystick signals and signal filtered with the BBF filter, (b) signals before the amplification, (c) output of the fuzzy system and conversion and integration of these results to be sent to the control board hardware.

from the filter BBF (signal $Z$ filt and $Y$ filt) are very satisfactory, proving the good performance of the filter in attenuating the noise components.

In Fig. 9(b), is represented the Ŷ-signal and Ẑ-signal after an adjustment of both signals. The Y-signal was inverted, amplified and is in the range of $[-1,1]$. The Z-signal was also amplified and converted to the range of $[-1,1]$.
Fig. 9(c) shows the two outputs of the fuzzy control system as well as the outcome of the smoothing process. Both signals were converted to the range of $[2.5,5]$ in order to be sent to the low-level control hardware of the DC motors (left and right).

The user starts its movement at $t=1 \mathrm{~s}$ and this intention is transmitted to the walker. The $\hat{Y}$-signal increases 
its amplitude (Fig. 9(b)), and this is interpreted as the user intention to go forward. Consequently, both fuzzy output signals increase their amplitude, as it can be seen in Fig. 9 (c) at $t \approx 1 \mathrm{~s}$, and the motors start to accelerate. The user keeps its movement until around $t \approx 9 \mathrm{~s}$.

At $t \approx 9 \mathrm{~s}$, the user wants to turn right until $t \approx 13 \mathrm{~s}$, Fig. 9(b), and consequently the $\hat{Z}$-signal increases its amplitude. This intention is captured by the proposed system and reflected in the fuzzy output signals: the right motor signal decreases its amplitude, and the left one maintains its. Therefore, the walker starts to turn right, as the user intends.

At $t \approx 16 \mathrm{~s}$, the user's intention is to stop, so he pushes the handles to himself. In Fig. 9(b) we can verify the amplitude of the $\hat{Y}$-signal ( $\hat{Z}$-signal) decreases (increases) accordingly. This happened because the user pushed the handles asymmetrically. However, this does not influence the movement of the walker, since we have implemented a safety rule which assures that the walker cannot move backwards. This is shown in Fig. 9(c) where at $t \approx 16 \mathrm{~s}$ the fuzzy output signals decrease to zero.

Despite the little variations of the $\mathrm{Y}$ and Z-signals, the motors present a constant and safe movement that correspond to the desired trajectories of the user, without bumps. Therefore, the system has been perfectly adjusted to read and infer the user's command intentions.

The device has been tested for different trajectories and the results were very encouraging. Further, the users were enquired about the driving of the device and the answer was that the vehicle was very manoeuvrable, easy to drive, and that it provided for a sense of security and was in synchrony with the desired movement.

\section{Conclusions}

This paper presented the first steps towards a methodology to extract the users' intent to guide the walker. The proposed interface sensor has been mounted onto the ASBGo walker and it is based on a joystick. A series of experiments with healthy users were performed which showed the sensibility of the joystick to acquire the navigation commands of the user.

Additionally, a control strategy that allows the walker to be driven according to the users intents was presented in this article. The proposed control system showed very good results, generating a control strategy with low computational cost, allowing a smooth and enjoyable driving, fast response of the walker and no sense of delay.

These results are very encouraging and have provided for a step further in the team aims of contributing for the design and improvement of new system architectures with low computational cost, and little and inexpensive embedded electronics.

However, these were the first steps and the next step is to validate the proposed system with elderly and disable people. Currently, the team is also including the encoders onto a closed loop control system which will provide for feedback about the walker actual state.

\section{Acknowledgments}

This work is financed by FEDER Funds and through "Programa Operacional Fatores de Competitividade" COMPETE and by National Funds through FCT - "Fundação para a Ciência e Tecnologia" under the Project: FCOMP-010124-FEDER-022674. Work supported by Portuguese Science Foundation (grant SFRH/BD/76097/2011).

\section{References}

[1] M. Martins, A. Frizera, C. Santos, R. Ceres, Assistive mobility devices focusing on smart walkers: classification and review, Robotics and Autonomous Systems (2011), http://dx.doi.org/10.1016/ j.robot.2011.11.015.

[2] G. Lacey, K. Dawson-Howe, Evaluation of Robot Mobility Aid for the Elderly Blind, in: Proceedings of the Fifth International Symposium on Intelligent Robotic Systems, 1997.

[3] A.C. Morris, R.R. Donamukkala, A. Kapuria, A. Steinfeld, J. Matthews J. Dunbar-Jacobs, S. Thrun, A robotic walker that provides guidance, in: Proceedings of the 2003 IEEE Conference on Robotics and Automation, May 2003, pp. 25-30.

[4] G. Lee, T. Ohnuma, N.Y. Chong, Design and control of JAIST active robotic walker. Intel Serv, Robotics, 2010.

[5] A. Frizera Neto, J.A. Gallego, E. Rocon, J.L. Pons, R. Ceres, Extraction of users navigation commands from upper body force interaction in walker assisted gait, BioMedical Engineering Online 9 (37) (2010).

[6] R. Ceres, J.L. Pons, L. Calderón, D. Mesonero-Romanos, V.F. Jiménez, X. Sánchez, P. Abizanda, B. Saro, G. Bonivardo, Andador activo para la rehabilitación y el mantenimiento de la movilidad natural. IMSERSO, Estudios I+D+I, 25:1-8, 2005

[7] H. Hashimoto, A. Sasaki, Y. Ohyama, C. Ishii, Walker with hand haptic interface for spatial recognition, In: Proceeding of the ninth IEEE International Workshop on Advanced Motion Control, May 2006, pp. $311-316$.

[8] A.J. Rentschler, R. Simpson, R.A. Cooper, M.L. Boninger, Clinical evaluation of Guido robotic walker, Journal of Rehabilitation Research Development 45 (9) (2008) 1281-1294.

[9] F. Shi, Q. Cao, C. Leng, H. Tan, Based On Force Sensing-Controlled Human-Machine Interaction System For Walking Assistant Robot in: Proceedings of the 8th World Congress on Intelligent Control and Automation, July 6-9 2010, Jinan, China, pp. 6528-6533.

[10] T. Renpeng, W. Shuoyu, J. Yinlai, I. Kenji, N. Masanori, Adaptive Controller for Motion Control of an Omni- directional Walker, in Proceedings of the 2010 IEEE International Conference on Mechatronics and Automation, (August 4-7, Xi'an, China) 2010, pp. 156-161.

[11] M. Alwan, A. Ledoux, G. Wasson, P. Sheth, C. Huang, Basic walkerassisted gait characteristics derived from forces and moments exerted on the walker's handles: Results on normal subjects, Med Eng Phys. 29 (3) (2007) 380-389.

[12] W. Gharieb. Intelligent Robotic Walker Design, in: International Conference on Automation, Robotics and Autonomous Systems, Sharm El Sheikh, Egypt, June 2006.

[13] E. Brookner, Tracking and Kalman Filtering Made Easy John Wiley and Sons, Inc., 1998.

[14] M. Grewal, A. Andrews 3rd, Kalman Filtering - Theory and Practice using Matlab, John Wiley \& Sons.

[15] E. Rocón, J. Ruiz, J. Moreno, J. Pons, A. Miranda, Tremor characterization: algorithms for the study of tremor time series, Sensors (2010).

[16] R.A. Kosinski, Literature Review on Reaction Time, Clemson University. Available from: <http://bsdc2009.pbworks.com/f/ literature+review+-+Clemson+Univ.pdf $>$.

[17] L.A. Zadech, Fuzzy Sets, Information, Control 8, 1965, pp. 338-353.

[18] Chris Kirtley, Clinical Gait Analysis: Theory and Practice, Churchill Livingstone, 2006. 